\title{
Psicoterapia do idoso: uma revisão da literatura
}

\author{
Roselisa Crespi Martins ${ }^{1}$ \\ Pontifícia Universidade Católica de Campinas
}

\begin{abstract}
O presente estudo visou levantar a produção científica referente à Psicoterapia do idoso, classificando-a em 06 dimensões de análise: países relacionados à pesquisa na área, os periódicos de indexação, os temas estudados, a abordagem utilizada, a modalidade de produção científica e os objetivos visados pelas pesquisas. O material analisado foi composto de 108 resumos de artigos de periódicos indexados ao PSYCHOLOGICAL ABSTRACTS (1990-1996), utilizando-se a base de dados PSYCLIT, com os verbetes de acesso: $p$ sychotherapy, brief psychotherapy, and: elderly, aging, geriatric, gerontology. Os resultados são discutidos em termos das dimensões propostas, levantando-se as tendências mais marcantes da produção nesse período e fazendo-se sugestões para o acúmulo de conhecimentos na área.

Palavras-chave: Psicoterapia do idoso, psicoterapia geriátrica, envelhecimento, gerontologia, idoso.
\end{abstract}

\begin{abstract}
Psychotherapy for older adults: a literature review

This paper examines the scientific literature concerning psychotherapy with older adults and classifies the studies on 06 dimensions: countries, periodics, themes, theoretical orientation, nature of the investigation and goals of researches. The sample was composed of 108 serials, selected from the PSYCLIT database (PSYCHOLOGICAL ABSTRACTS, from 1990 to 1996) using the following key words: psychotherapy, brief psychotherapy, and: elderly, aging, geriatric, gerontology. Results are discussed according to the analytical dimensions proposed, the researche main trends are identified and suggestions are presented to improve new studies in the area.

Key words: Psychotherapy for older adults, geriatric psychotherapy, geriatrics, gerontology, aged, elderly.
\end{abstract}

\section{Introdução}

Nas últimas décadas, o estudo do envelhecimento, situado na interface entre as ciências biológicas, sociais e humanas, vem assumindo um interesse crescente. $\mathrm{Na}$ maioria dos países o aumento da expectativa de vida vem justificando os investimentos sociais e a adoção de políticas e práticas que considerem as demandas da popula-

1. Mestre em Psicologia Clínica pela PUC-Campinas. Endereço para correspondência: Rua Capivari, 267, Jd. Novo Campos Elíseos, CEP13050-571,Campinas, SP, E-Mail: roselisa@uol.com.br ção idosa, em termos assistenciais, educacionais, ocupacionais, recreacionais, de saúde, de previdência e outros.

Concomitantemente, verifica-se um verdadeiro "boom"do trabalho científico voltado a essa área de investigação. O envelhecimento, um assunto complexo, vem, curiosamente, revolucionando conceitos, forçando a revisão de posicionamentos teóricos e imprimindo novo dinamismo a todos os campos do saber. Nas ciências naturais, por exemplo, questiona-se o conceito de doenças "da idade", dado que enfermidades como osteoartrites, diabetes, 
demência, e outras, tradicionalmente assim consideradas, não são exclusivas dessa faixa etária, devendo ser vistas como processos patológicos como quaisquer outros de que o ser humano possa ser acometido em seu ciclo vital (Laks,1995). A associação entre idade e declínio cognitivo, igualmente, vem sendo revista, sendo que algumas pesquisas apontam melhorias no exercício de algumas atividades intelectuais em idosos após os 65 anos (Stern \& cols,1994). As ciências sociais, dentre várias contribuições, vêm demonstrando, a partir do estudo das representações sociais, como socialmente se constroem significados sobre o envelhecimento (Santos,1994), ou ainda, o quanto se compartilham estereótipos e preconceitos, encerrando os idosos em um grupo de referência negativa (Lopes,1990). E quanto à ciência psicológica, ressaltam-se aqui duas de suas contribuições: uma, de caráter teórico, conduzindo a inovações numa das pedras angulares da Psicologia: o conceito de desenvolvimento; e outra, objeto deste artigo, com respeito às práticas psicoterápicas.

Segundo Neri $(1993,1995)$, na visão tradicional, o desenvolvimento era visto de forma linear e unidirecional, na forma de aquisições sucessivas rumo à maturidade. Os pesquisadores da vida adulta e da velhice questionaram esse reducionismo, resultando numa perspectiva de desenvolvimento multidirecional e multidimensional. Nessa nova perspectiva, o desenvolvimento é considerado um processo que envolve equilíbrio dinâmico entre perdas e ganhos, sobreposição de aspectos que se modificam em ritmos diferentes, pluralismo na direção das mudanças (podendo haver, por exemplo, crescimento num sistema e declí- nio em outro) e uma grande heterogeneidade (variabilidade intra-individual no desenvolvimento, dependendo da plasticidade e das potencialidades). Dentro desse enfoque, denominado de curso de vida, o desenvolvimento é tido como resultante da interação dialética de três sistemas: gradação por idade (processos de maturação biológica e de socialização), contexto histórico (fatores biológicos e ambientais que afetam grupos inteiros no tempo histórico) e eventos não-normativos (que não têm caráter universal, nem são previsíveis, como nos eventos idiossincráticos, do tipo: divórcio, acidente, doença repentina etc.). E ainda, nessa proposição, o desenvolvimento passa a ser considerado como multifacetado e multideterminado, pressupondo seu enfoque como área multidisciplinar, que toma em conta a contribuição integrada de disciplinas, tais como a biologia, a sociologia, a antropologia e a psicologia.

Como decorrência dessa nova concepção, pode-se entender a formação de perfis vitais bastante diferenciados de uma pessoa para outra, dado que o desenvolvimento não se traduz em mudanças cumulativas e unidirecionais, e sim, em mudanças descontínuas e multilineares. A não ser pela heterogeneidade, torna-se difícil definir queméo idoso, como se apresenta e se situa no interjogo sutil da multiplicidade de todos esses fatores intervenientes.

Tomando-se em consideração o campo das psicoterapias, constatam-se esforços no sentido de revisar posicionamentos teóricos e de ajustar técnicas e estratégias para o atendimento clínico a idosos. 
Essa tendência se faz sentir de forma ampla, com relação às modalidades das diversas orientações teóricas.

A psicanálise, por exemplo, tradicionalmente não se aplicaria aos idosos devido à pouca plasticidade dos processos mentais (Freud, 1904-1905). Hoje, no entanto, a orientação psicanalítica é amplamente adotada, constando contribuições bastante valiosas (Radebold,H.,1994) e que favorecem o entendimento de questões como a identidade, a auto-imagem e os processos de luto nos idosos.

Ainda, dentro da abordagem psicodinâmica, Braier (1984) recomenda para o tratamento dos idosos a modalidade técnica da psicoterapia breve, considerando que a mesma permite trabalhar setorialmente, respeitando a estrutura caracteriológica do paciente e evitando mobilizações afetivas desnecessárias e arriscadas.

Ao examinar a literatura concernente ao tratamento de idosos, pode-se constatar que artigos de revisão (Krebs, 1990) e de metanálise (Scogin \& McElreath, 1994; Schneider,1994) são bastante incomuns. Krebs examina o campo das psicoterapias de idosos, segundo seis categorias de análise: a) os objetivos das psicoterapias, b) as teorias de desenvolvimento, c) as situações críticas para o idoso, d) as diferenças de gênero e suas implicações no tratamento, e) o papel do psicoterapeuta e f) os diferentes tipos de psicoterapias (individual, casal, grupo), enfatizando, de modo geral, a necessidade de maior número de pesquisas que investiguem a eficácia das diversas modalidades psicoterápicas. Já, em metanálise, citam-se duas pesquisas, ambas relativas à depressão em idosos: Scogin \&
McElreath, que comparam resultados de diversas modalidades psicoterápicas (comportamental, cognitiva, psicodinâmica, de reminiscência e eclética) e Schneider, que investiga o efeito de medicamentos antidepressivos. Mais atinente aos interesses da Psicologia, o artigo de Scogin \& McElreath examina um período de 15 anos de práticas psicoterápicas junto a idosos (entre 1975 e 1990), concluindo pela validade de todas as modalidades de intervenção, sem evidências de superioridade de quaisquer umas sobre as outras.

$\mathrm{Na}$ presente revisão empreende-se um exame dos artigos sobre psicoterapias de idosos, como formas científicas de construção de um conhecimento. Trata-se de um levantamento, com pretensões de uma primeira aproximação ao tema, com vistas a contribuir por situar minimamente os interessados em uma panorâmica atual das tendências das pesquisas na área.

\section{Método}

\section{Material}

Resumos de artigos de periódicos indexados ao Psychological Abstracts, no período compreendido entre 1990 e 1996, referentes a psicoterapias realizadas com adultos acima de sessenta anos. Segundo a Organização Mundial de Saúde (OMS) são considerados idosos os indivíduos acima de 65 anos, vivendo em países desenvolvidos, ou acima dos 60, no caso dos que viviam em países subdesenvolvidos ou em desenvolvimento. Foram considerados os indíviduos acima de 60 anos, visando a obtenção da maior amostra possível para o exame da literatura no âmbito internacional. 


\section{Procedimento}

Primeiramente, foi conduzida uma pesquisa em computador, utilizando-se a base de dados PSYCLIT, com os verbetes de acesso: psychotherapy, briefpsychotherapy, and: elderly, aging, geriatric, gerontology.

A seguir, foram separadas as referências relativas a adultos acima de sessenta anos.

Dessa pesquisa resultaram 108 resumos de artigos, publicados entre 1990 e 1996, que foram então submetidos a seis dimensões de análise, de acordo com:

1. Periódico de divulgação.

2. País de origem da pesquisa .

3. Tema estudado.

4. Abordagem: modalidade de atendimento (individual, grupo, conjugal, familiar, breve, apoio, interpessoal, construtivista, corporal, psicofarmacológica, outra), incluindo o enfoque teórico (psicodinâmico, comportamental, cognitivista, sistêmico, construtivista, fenomenológico, múltiplo) sempre que possível.

5. Modalidade científica: tipo de produção científica que originou o artigo (pesquisa, comunicação da prática, discussão teóricoprática, discussão teórica).

6. Objetivo da pesquisa: diagnóstico, processo, resultado, follow-up, múltiplo.

\section{Resultados}

Os dados foram apresentados em termos de porcentagem de ocorrência com relação às seis dimensões de análise utilizadas para o exame da produção científica na área.
Na Tabela 1 foram destacados os periódicos mais utilizados na divulgação dos estudos em psicoterapia do idoso.

Tabela 1. Distribuição de artigos em psicoterapia de idosos por periódico de indexação.

\begin{tabular}{|c|c|}
\hline Periódico & Porcentagem \\
\hline Generations & $6,48 \%$ \\
\hline Psychotherapy & $5,55 \%$ \\
\hline Zeitsclirift fur Gerontologie & $4,62 \%$ \\
\hline
\end{tabular}

Dois periódicos de língua inglesa se evidenciaram com o maior número de publicações: Psychotherapy e Generations, seguindo-se das publicações em alemão Zeitschrift fur Gerontologie.

Os EUA figuraram como os mais especializados na matéria, respondendo pela maior parte das publicações, seguindo-se da Alemanha ( Tabela 2 ).

Tabela 2. Distribuição de artigos em psicoterapia de idosos por país de origem da produção.

\begin{tabular}{|c|c|}
\hline País & Porcentagem \\
\hline EUA & $60,18 \%$ \\
\hline Alemanha & $15,74 \%$ \\
\hline Canadá & $3,70 \%$ \\
\hline
\end{tabular}

A Tabela 3 é ilustrativa dos temas vinculados aos estudos em psicoterapias de idosos. 
Tabela 3. Distribuição de artigos em psicoterapia de idosos por temas pesquisados.

\begin{tabular}{|l|c|}
\hline \multicolumn{1}{|c|}{ Temas } & Porcentagem \\
\hline Depressão & $20,37 \%$ \\
\hline $\begin{array}{l}\text { Avaliação das Psicoterapias } \\
\text { Geriátricas }\end{array}$ & $9,25 \%$ \\
\hline $\begin{array}{l}\text { Aspectos das Psicoterapias } \\
\text { Geriátricas }\end{array}$ & $6,48 \%$ \\
\hline $\begin{array}{l}\text { Perspectivas das Psicotera- } \\
\text { pias Geriátricas }\end{array}$ & $6,48 \%$ \\
\hline Estratégias Terapêuticas & $4,62 \%$ \\
\hline Desordens Psicossomáticas & $3,70 \%$ \\
\hline Suicídio & $3,70 \%$ \\
\hline Desordens do Stress & $2,77 \%$ \\
\hline
\end{tabular}

A psicoterapia de idosos, com maior frequência, apareceu associada ao diagnóstico de depressão ou depressão maior, evidenciando-se resultados bem-sucedidos mediante o emprego de diversas modalidades psicoterapêuticas (interpessoal, adleriana, psicodinâmica, cognitiva, individual, grupo, breve, psicofarmacológica, dentre outras).

A seguir, na categoria "avaliação das psicoterapias geriátricas", evidenciaramse estudos de mensurações da eficácia das diversas modalidades de atendimento, estudos esses conduzidos segundo metodologias diversificadas. Por exemplo, Arefjord (1994) procurou avaliar a eficácia dos atendimentos psicológicos a idosos mediante questionários enviados a psicoterapeutas experientes. Peters \& Lange (1994) se utillizaram de questionários, porém dirigidos aos idosos em situação de followv-up, que informaram sua visão dos resultados obtidos na terapia e o grau de satisfação com a mesma. Já Danilov \& Selivra
(1990) utilizaram-se de um delineamento de pesquisa para investigar a eficácia da terapia familiar, no qual foram comparados resultados de pacientes idosos de diversos quadros clínicos entre si e com um grupo controle. Fretter et al (1994), trabalhando com psicoterapia breve, procuraram investigar as relações existentes entre três variáveis: os progressos do paciente idoso, o grau de adequação das intervenções do terapeuta e a compatibilidade de tais intervenções ao plano terapêutico estabelecido.

Seguiram-se estudos acerca dos "aspectos das psicoterapias", envolvendo o que há de mais específico com relação ao atendimento a idosos (os temas mais comuns, as dinâmicas transferenciais e contratransferenciais envolvidas, critérios para a indicação da psicoterapia, manejo do término do atendimento etc). Ilustrando, Chodorkoff (1990) explorou os problemas típicos dessa clientela e questões relacionadas à seleção de uma modalidade de tratamento dentre as psicoterapias, as psicofarmacoterapias e as abordagens educacionais.

Vieram, a seguir, estudos sobre as principais tendências (ou "perspectivas"), apontando as áreas que vêm assumindo importância crescente e sinalizando as prioridades em termos de novos conhecimentos. Dentre os diversos trabalhos, citase, por exemplo, Radebold (1992), que enfatizou a necessidade de estender aos idosos a mesma gama de atendimentos psicológicos acessíveis aos adultos mais jovens. Outro pesquisador, Heuft (1990) discutiu as tendências das psicoterapias psicanalíticas com idosos, concluindo que as mesmas devam incorporar as perspecti- 
vas da nova gerontologia psicossomática, considerando a complexa interação do fisiológico, do psicológico e do fator social.

A Tabela 4 apresenta a distribuição das terapias em função da abordagem teórica.

Tabela 4. Distribuição de artigos em psicoterapias de idosos com relação à abordagem ou modalidade psicoterapêutica empregada.

\begin{tabular}{|l|c|}
\hline \multicolumn{1}{|c|}{ Abordagem } & Porcentagem \\
\hline Múltipla & $25,00 \%$ \\
\hline Psicoterapia Psicodinâmica & $14,81 \%$ \\
\hline Psicoterapia Breve & $12,03 \%$ \\
\hline Integrada & $11,11 \%$ \\
\hline Psicotérapia Psicanalítica & $8,33 \%$ \\
\hline Psicoterapia de Grupo & $8,33 \%$ \\
\hline Psicoterapia Interpessoal & $4,62 \%$ \\
\hline Psicoterapia de Apoio & $2,77 \%$ \\
\hline Psicoterapia Comportamental & $2,77 \%$ \\
\hline
\end{tabular}

Com base na Tabela 4, verificou-se que a maior parte dos estudos $(25 \%)$ investigou uma variada gama de procedimentos empregados junto ao paciente idoso. Em tais artigos, nos quais diversas (ou "múltiplas") abordagens teóricas foram examinadas, discutiram-se, por exemplo, critérios de indicação, aspectos técnicos ou teóricos, efetuando-se comparações entre várias modalidades psicoterápicas com vistas à avaliação de sua eficácia. Exemplificando, Heuft (1993), com base em atendimentos realizados com 20 idosos, discute a indicação e o alcance da psicanálise de longo termo, da psicoterapia analítica e da psicoterapia focal no tratamento das desordens psicossomáticas.
Cabe ainda destacar que, embora nas psicoterapias geriátricas tenha se observado a tendência à utilização das modalidades usuais de tratamento ao adulto, também se verificou o emprego de modelos específicos para o idoso (como o de revisão-de-vida e psicoterapia de reminiscência), registrando-se ainda a presença de abordagens menos tradicionais (como a terapia da dança e do movimento, técnicas de meditação, a psicoterapia computadorizada etc.). Petzold (1991) descreveu três casos bem-sucedidos de pacientes idosos tratados mediante técnicas como a dança e o movimento; Hyer et al (1995) estudaram a influência do stress pós-traumático em veteranos de guerra, incluindo em sua abordagem a psicoterapia cognitivo-comportamental, a dessensibilização e a reminiscência; Callanan (1994) se utilizou da arteterapia para mobilizar o imaginário pessoal de idosos, favorecendo sua expressão autêntica, criatividade e a resolução de conflitos; Halbrook \& Duplechin (1994) discutiram o toque como intervenção em psicoterapia corporal com idosos, refletindo sobre seu significado afetivo e cultural e explanando a potencialidade desse recurso terapêutico.

Outros $11,11 \%$ dos trabalhos se aplicaram a investigar diversas modalidades, porém empregadas de maneira conjunta. Nessas terapias, aqui denominadas "integradas", várias modalidades foram associadas de forma a compor uma única terapêutica. Foram investigados, por exemplo, os efeitos de associações entre psicoterapia e farmacoterapia, psicoterapia e técnicas de relaxamento, psicoterapia individual concomitante com terapia de gru- 
po, familiar, conjugal, reabilitação etc. Ilustrando, Ionedes (1992) apresentou um programa terapêutico para pacientes com Alzheimer, incluindo: medicação, psicoterapia individual, psicoterapia de grupo, psicodrama e aconselhamento familiar. Por vezes, verificou-se tal integração com relação a uma mesma abordagem teórica: Sperry (1992) integrou os princípios de quatro perspectivas da psicoterapia adleriana (psicodinâmica, cognitiva/comportamental, sistêmica e experimental) no tratamento a pacientes idosos; Leszcz (1990) propôs um modelo que associa três abordagens da psicoterapia de grupo (psicodinâmica, cognitiva/comportamental e desenvolvimentista), obtendo bons resultados junto a idosos deprimidos.

Observou-se ainda que, na composição dos tratamentos integrados, prevaleceu a associação clássica entre uma intervenção psiquiátrica medicamentosa $\mathrm{e}$ alguma (ou algumas) modalidade(s) de intervenção psicológica, como em Reynolds et al (1992).

No tocante às abordagens estudadas individualmente, evidenciou-se alguma concentração de artigos na orientação psicodinâmica $(14,81 \%)$, como o estudo de Bron (1992), conduzido com 155 pacientes acima de 60 anos, diagnosticados segundo três tipos de depressão (endógena, neurótica e reativa), que discutiu aspectos psicodinâmicos das reações de luto patológicas e de outros sintomas que compõem a síndrome das doenças depressivas da velhice. Seguiram-se $12,03 \%$ das publicações em psicoterapias breves, dentre as quais Winston et al (1991) que abordaram 32 idosos segundo duas técnicas breves (psicodi- nâmica e adaptativa), verificando semelhanças e diferenças obtidas em medidas de processo e de resultado. Seguiram-se os artigos em terapias de grupo e psicanalíticas $(8,33 \%$ cada), interpessoais $(4,62 \%)$, de apoio e comportamentais ( $2,77 \%$ cada).

Quanto às modalidades de produção científica (Tabela 5) que originaram os artigos, se dividiram, principalmente, entre pesquisas $(40,75 \%)$ e discussões teóricas (38,88\%), seguindo-se de estudos teóricopráticos $(17,59 \%)$ e de comunicações da prática $(2,78 \%)$.

Tabela 5. Distribuição de artigos em psicoterapia de idosos em função da modalidade de produção científica que os originou

\begin{tabular}{|l|c|}
\hline \multicolumn{1}{|c|}{ Modalidade científica } & Porcentagem \\
\hline Pesquisas & $40,75 \%$ \\
\hline Estudos teóricos & $38,88 \%$ \\
\hline Discussões teórico-práticas & $17,59 \%$ \\
\hline Conmunicações da prática & $2,78 \%$ \\
\hline TOTAL & $100 \%$ \\
\hline
\end{tabular}

Dentre as contribuições já referidas, algumas se constituem exemplos de delineamento de pesquisa, como Arefjord (1994), Danilov \& Selivra (1990), Peters \& Lange (1994), Fretter et al (1994), dentre outras. Estudos teórico-práticos, nos quais, no geral, uma discussão teórica é ilustrada com um ou mais estudos-de-caso, puderam ser observados, por exemplo, em Chodorkoff (1990) e Callanan (1994). Discussões teóricas foram encontradas em contribuições como o de Radebold (1992). Comunicações da prática, no geral se referindo a relatos de experiências desenvolvi- 
das em programas de saúde mental em instituições, são ilustradas por Lustbader (1990), que apresentou um modelo associativo entre psicoterapia individual, familiar e de grupo oferecido em um centro comunitário de saúde mental para idosos.

Dentre as pesquisas conduzidas em psicoterapias de idosos (40,75\% dos artigos), a maior parte teve por objetivo a investigação de processo, seguindo-se da investigação ligada a resultados, depois a followv-up e por último, a diagnóstico (Tabela 6).

Tabela 6 - Distribuição de artigos em relação aos objetivos das pesquisas.

\begin{tabular}{|l|c|}
\hline \multicolumn{1}{|c|}{ Objetivos das pesquisas } & Porcentagem \\
\hline Processo & $18,52 \%$ \\
\hline Resultado & $12,04 \%$ \\
\hline Processo e Resultado & $5,56 \%$ \\
\hline Resultado e Follozv-up & $3,71 \%$ \\
\hline Diagnóstico e Processo & $0,92 \%$ \\
\hline TOTAL & $40,75 \%$ \\
\hline
\end{tabular}

\section{Discussão}

Os dados permitiram evidenciar os EUA, seguidos pela Alemanha, como os dois países de maior expressão no tocante à produção científica na área.

Quanto aos temas, a depressão sobressaiu-se como o mais estudado quando se enfoca a população de idosos. A depressão é apontada, na literatura, unanimemente, como a psicopatologia mais expressiva do grupo de idosos. Sua alta incidência nesse grupo pode ser entendida, dentro do moderno enfoque da Gerontologia, não como "uma doença da velhice", mas como expressão de um fenômeno mais amplo de desajustamento a essa etapa do curso de vida.

Vêm sendo bastante estudados, também, os temas voltados às psicoterapias geriátricas em si, explorando-se, por exemplo, questões típicas, tarefas e desafios concernentes ao atendimento de idosos, critérios para a escolha da modalidade terapêutica (psicofarmacológica, psicoterápica etc.), a formação e a experiência clínica necessárias ao psicoterapeuta, e ainda, as avaliações da eficácia das diversas técnicas e estratégias psicoterápicas, tomadas individualmente ou associadas. Além desses temas, mais comuns, qualitativamente também se verificou uma pulverização do campo de estudo, abrangendo uma ampla gama de assuntos, como por exemplo, as desordens psicossomáticas, o suicídio, o stress, o luto, a ansiedade, as psiconeuroses etc.

Uma consideração importante com relação à psicoterapia de idosos é a de que a maior parte dos estudos tem se dedicado a examinar a eficácia das diversas técnicas psicoterapêuticas, constatando-se resultados bem-sucedidos em todas as modalidades. Tais dados sugerem, portanto, que os problemas psicológicos dos idosos possam ser abordados, com sucesso, de forma tão variada quanto os de outros adultos. Também tem sido destacada a utilidade do emprego integrado de diversas modalidades em um único programa de tratamento, o que está coerente com a concepção multifacetada e interdisciplinar do envelhecimento. A observação de que a maior parte das propostas parece associar a farmacoterapia a algum(ns) tipo(s) de intervenção psicológica possivelmente se ligue ao tipo de tema 
prevalente nessa literatura, ou seja, a psicoterapia de idosos com depressão maior ou outros quadros clínicos de depressão.

Em suma, o exame do conjunto dessa produção permite evidenciar uma tendência ao enfoque teórico múltiplo e à abordagem multidisciplinar. Mesmo no campo das psicoterapias, dentro de uma mesma modalidade, procura-se incorporar princípios derivados de diversas orientações teóricas. Procura-se, com isso, construir um modelo mais integrativo, que possa melhor prover as necessidades do atendimento ao idoso.

$\mathrm{Na}$ presente revisão, os estudos se dividiram, com respeito à forma de produção de conhecimento, entre pesquisas e discussões teóricas, com alguma predominância das pesquisas. No entanto, quando se observa que, aos estudos teóricos, se seguem as discussões teórico-práticas, que, no geral, se valem da descrição de um caso único como ilustração da teoria em discussão, a "balança" então se inclina em direção contrária às pesquisas. Tomadas em conjunto, as discussões teóricas e de cunho teórico-prático, totalizando $56,47 \%$ das publicações, podem sugerir a necessidade de uma maior sistematização do conhecimento que vem sendo produzido na área.

E ainda, como uma última observação proveniente dos dados, quando pesquisas são realizadas, verifica-se que a maior parte delas investiga processo e resultado, sendo ainda incipientes na área os estudos de acompanhamento e, mais ainda, os que investigam diagnóstico.

Enfatiza-se a necessidade de outros estudos de revisão de literatura, que se constituem, sem dúvida, numa ferramenta de grande utilidade para os pesquisadores e profissionais voltados a uma área que se mostra em franca expansão científica, como a psicoterapia de idosos.

Quanto a este estudo, sugere-se sua ampliação, incluindo-se outras palavraschave além de psychotherapy, como o termo inglês counseling, que parece ser também bastante expressivo para caracterizar o atendimento psicológico a idosos. Como no Brasil e em outros países latino-americanos, pouco se publica nos Psychological Abstracts, os quais serviram de base à presente pesquisa, estudos semelhantes poderiam ser conduzidos junto a bases de dados de língua latina para um maior conhecimento do estágio atual das pesquisas gerontológicas em nosso meio.

Conclui-se, acreditando que uma das principais contribuições da Ciência é, indubitavelmente, a mudança de mentalidade. A investigação do envelhecimento vem, aos poucos, fazendo com que o mesmo deixe de ser sinônimo de doenças, de perdas e de disfunções, rumo a uma consideração mais ampla, tomada dentro de uma perspectiva integracionista e desenvolvimentista de curso de vida.

A psicoterapia do idoso representa o desafio de um campo novo e promissor, no qual toda a bagagem do psicoterapeuta experiente pode ser útil, qualquer que seja a modalidade clínica e a orientação teórica por ele abraçada. Faz parte da aventura de todo bom viajante, no entanto, a disposição a ampliar um tanto mais sua bagagem, na medida em que isso se revele enriquecedor e necessário, bem como a abrir mão de alguns itens que, comprovadamente, têm se revelado sem serventia. A mesma disposi- 
ção a fazer adaptações, que o terapeuta demonstra quando assume seus pacientes (por exemplo, crianças ou adolescentes) tomando em consideração seu curso de vida, aqui também deve estar presente. Para tanto, a literatura aí está, a nos auxiliar, farta e disponível...

\section{Referências Bibliográficas}

Arefjord, K. Psychotherapy and the elderly: a study emphasizing need, supply and demand. Tidsskrift for Norsk Psykologforening, 1994, 31 (10): 919-924.

Braier, E.A. (1984). Psicoterapia Breve de orientação psicanalítica. São Paulo: Martins Fontes.

Bron, B. Psychotherapy of depressive aging patients with pathological grief reactions. Zeitschrift fur Psychosomatische Medizin und Psychoanalyse, 1992, 38 (4): 346-357.

Callanan, B.O. Art therapy with the frail elderly. Journal of Long Term Home Health Care: the PRIDE Institute Journal, 1994, 13 (2): 20-23.

Chodorkoff, B. Providing psychotherapy in private practice. Special Issue: Counseling and therapy for elders. Generations, 1990, 14 (1): 27-30.

Danilov, Y.M \& Selivra, E.A. The problem of avaliation of the family interrelationsips and the efficacy of psychotherapy for elderly patients. Trudy Leningradskogo Nauchno-Issledovatel' skogo Psykhonevrologicheskogo Instituta im V M Bekhtereva, 1990, 125 : 97-103.

Fretter, P.B.; Bucci,w.; Broitman, J.; Silberschatz, G. et al. How the patient's plan relates to the concept of transference. Psychotherapy Research, 1994, 4 (1): 58-72.

Freud, S.(1904-1905). Sobre a psicoterapia. In: Obras Completas, VII Rio de Janeiro: Imago, 1969: 267-278.

Halbrook, B. \& Dupliichin, R. Rethinking touch in psychotherapy: guidelines for practio- ners. Psychotherapy in Private Practice, 1994, 13 (3): 43-53.

Heuft, G. Future research perspectives in psychoanalytically-oriented psychosomatic gerontology: personality and the aging process. Zeitshcrift fur Gerontologic, 1990, 23 (5): 262-266.

Heuft, G. Psychoanalytic gerontopsychosomatics: genesis and differential psychotherapy indication of acute functional somatisations in the elderly. Psychotherapie Psychosomatik Medizinische Psychologie, 1993, 43 (2): 46-54.

Hyer, L.; Summers, M.N.; Braswell, L.; Boyd, S. Posttraumatic stress disorder: silent problem among older combat veterans. Psychotherapy, 1995, 32 (2): 348-364.

Ionedes, N.S. A therapy program for Alzheimer's disease. Special Issue: the process of aging and working with older adults. Individual Psychology: Journal of Adlerian Theory Research E Practice, 1992, 48 (4): 413-418.

Krebs, E. Psychotherapy in old age. Schweizer Archiv fur Neurologie und Psychiatrie, 1990, 141 (4): 361-380.

Laks, J. O que há de tão especial em ter 65 anos? Journal Brasileiro de Psiquiatria, 1995, 44 (7): 341-343.

Leszcz, M. Towards an integrated model of group psychotherapy with the elderly. International Journal of Group Psychotherapy, 1990, 40 (4): 379-399.

Lopes, R.G.C. Investigação a respeito do projeto de vida de idosos que se mantém socialmente ativos. Dissertação de Mestrado, São Paulo, PUC, 1990.

Lustbader, W. Mental health services in a community health center. Special Issue: counseling and therapy for elders. Generations 1990, 14 (1): 22-23.

Neri, A. L. (Org.) - (1993) Qualidade de vida e idade madura. Campinas: Papirus. (1995) - Psicologia do envelhecimento. Campinas: Papirus.

Peters, M. \& Lange, C. Follow-up study in elderly patients on the effects of inpatient psychothe- 
rapy. Zeitschrift fur Gerontopsychologie $\mathcal{E}$ Psychiatrie, 1994, 7 (1): 47-56.

Petzold, H.G. Integrative dance and motion therapy in treating aged persons. Praxis der Psychotherapie und Psychosomatik, 1991, 36 (4): 195-206.

Psyclit: Journal articles 1990 Jun.1996 [CDROM]. Washington, American Psychological Association/Silver Platter, 1996.

Radebold, H. Goals of psychotherapy for the elderly. Zeitschrift fur Gerontologie, 1992, 25 (6): 349-355.

Radebold, H. Psychoanalytic psychotherapy and psychoanalysis with elderly people. Zeitschrift fur Psychoanalytische Theorie und Praxis, 1994, 9 (4): 439-451.

Reinolds, C.F.; Frank, E.; Perel, J.M.; Imber, S.D. et al. Combined pharmacotherapy and psychotherapy in the acute and continuation treatment of elderly patients with recurrent major depression: a preliminary report. American Journal of Psychiatry, 1992, 149 (12): 1687-1692.

Santos, M.F.S. Velhice: uma questão psico-social. Temas em Psicologia, 1994, 2: 123-131.

Schneider, L,S. (1994) Meta-analysis from a clinician's perspective. In: L.S. Schneider \& cols, Diagnosis and treatment of depression in late life: results of the NIH Consensus Development Conference. Washington (DC): American Psychiatric Press.

Scogin, F. \& Mcelreath, L. Efficacy of psichosocial treatments for geriatric depression: a quantitative review. Journal of Consulting and Clinical Psychology, 1994, Vol.62 (l): 699-74.

Sperry, L. Psychotherapy systems: an Adlerian integration with implication for older adults. Special Issue: The process of aging and working with older adults. Individual Psychology: Journal of Adlerian theory, research E practice, 1992, 48 (4):451-461.

Stern, R.G. et al A longitudinal study of Alzheimer's disease measurement, rate, and predictors of cognitive deterioration. American Journal of Psychiatry, 1994, 151: 390-396.
Winston, A.; Pollak, J.; Mccullough, L.; Flegenheimer, W. et al. Brief psychotherapy of personality disorders. Journal of Neroous $\mathcal{E}$ Mental Disease, 1991, 179 (4): 188-193. 